\title{
Influence of foot progression angle on center of pressure during stair ascending in subjects with chronic ankle instability
}

\author{
Jung-Hyun Ban ${ }^{1}$, Tae-Ho Kim² \\ ${ }^{1}$ Department of Physical Therapy, Graduate School of Daegu University, Daegu, Republic of Korea \\ (bjh0760@gmail.com) \\ ${ }^{2}$ Department of Physical Therapy, Daegu University, Daegu, Republic of Korea \\ (ptkimth@daegu.ac.kr) \\ Corresponding author : Tae-Ho Kim (ptkimth@daegu.ac.kr)
}

Received: February 20, 2021. Revised: July 8, 2021. Accepted: August 3, 2021. Published: August 11, 2021.

\begin{abstract}
The purpose of this study was to identify changes in the center of pressure during stair ascending in subjects with chronic ankle instability while different angles of foot are applied.

The subjects of this study were 20 male and female adults with chronic ankle instability were selected from among the employees of D Hospital in Daegu Metropolitan City. The criteria for selection of subjects with chronic ankle instability were those who felt wobbling in the ankle joint and scored not higher than 24 points in a test using the Cumberland Ankle Instability Tool (CAIT). The subjects carried out stair ascending in neutral, toe-in and toe-out postures, respectively, and changes in the center of pressure (COP) were compared and analyzed.

The results of this study, no statistically significant difference appeared in the comparison between the toe-in posture and neutral posture or between the neutral posture and the toe-out posture but medial/lateral movements of the center of pressure showed significant differences between the toe-in and toe-out postures. In addition, the total travel range and the moving range of the center of pressure, the average velocity, and the anterior/posterior movements of the center of pressure showed no statistically significant difference among all three postures.

As a result, it could be seen that when adults with chronic ankle instability climb the stairs, the toe-in posture reduce the medial/lateral movements of the center of pressure thereby increasing the stability of the ankle and effectively preventing re-injuries.
\end{abstract}

Keywords: ankle instability, foot progression angle, stair ascending, center of pressure, ankle posture.

\section{INTRODUCTION}

The ankle joint is one of the representative lower limb joints, plays a very important role in absorbing shock and carrying out normal walking, and is one of those joints where orthopedic injuries occur the most frequently not only in the case of athletes but also in the case of ordinary people [1]. As for the range of motion of the ankle joint, the range of inversion becomes larger than the range of eversion because the lateral malleolus of the fibula is located below the medial malleolus of the tibia [2]. Due to this structural characteristic, injuries of the ligament of the ankle joint are mainly caused during inversion among the movements of the ankle joint, and lateral ligaments representatively including the anterior talofibular ligament, the calcaneofibular ligament, and the posterior talofibular ligament are mainly injured. According to a study conducted by [3], injuries to the ligaments in the ankle area can be functionally healed to some extent only with appropriate conservative therapy in the early stage, but since protection and treatment are not properly carried out according to the degree of injuries, repetitive re-injuries occur in about $20-40 \%$ of patients and such re-injuries spread into chronic ankle instability [3].

The main cause of chronic ankle instability is anatomical instability. Since the lateral ligament, which is relatively weaker than the medial ligament, is mainly injured when an ankle has been sprained, the anterior dislocation of the fibula can be a contributing factor to ankle instability [4].

In general, chronic ankle instability is classified into functional ankle instability and mechanical ankle instability. With regard to functional ankle instability, it has been reported that when the muscles, ligaments and tendons supporting regions around the ankle have not been recovered into their previous normal state due to insufficient rehabilitation after an ankle injury, symptoms such as weakening of muscle strength, declines of balance and coordination abilities, etc. appear, and these symptoms cause ankle joint instability or repetitive injuries in subjective forms [5]. On the other hand, mechanical ankle instability shows objective forms such as joint motions exceeding the normal range of motion measured with radiological or clinical evaluation or the laxity or rupture of ligaments such as the anterior talofibular ligament, the posterior talofibular ligament, and the calcaneofibular ligament [2], and it has been reported that pathological laxity such as chronic ankle instability leads to postural control disorder, defects in the muscle nerve reflexes of the lower limb muscles, as well as 


\begin{tabular}{lcc}
\hline Subjects & $\begin{array}{r}\text { adults with chronic } \\
\text { ankle instability }\end{array}$ & $\boldsymbol{p}$ \\
\hline Age(year) & $29.55 \pm 2.04$ & .07 \\
Sex(male/female) & $12 / 8$ & .725 \\
Height $(\mathrm{cm})$ & $168.25 \pm 6.06$ & .461 \\
Weight $(\mathrm{kg})$ & $61.95 \pm 10.83$ & .58 \\
Foot length(mm) & $21.90 \pm 3.78$ & .673 \\
\hline
\end{tabular}

mean \pm standard deviation

${ }^{*} \mathrm{p}<.05$

defects in lower extremity muscle strength, the motor sensation, and the joint control sensation [6].

In the case of subjects with chronic ankle instability, whose lower extremity muscle strength has been weakened and proprioceptive sensibility has declined as such, the ability to perform and balance ability are reduced when they walk on flatlands or especially when they walk on stairs so that the risk of falls increases [7].

Stair walking is a movement that occurs the most frequently next to flatland walking in our daily life [8]. Studies reported that in stair ascending movements [9], larger ranges of motion and moments of flexion and extension appear in the lower limb joints compared to flatland walking, indicating that higher muscle strength and force are required during stair ascending stairs [10]. The high risks of injuries during stair walking as such are also closely associated with damage to the overall lower limb joints such as the knee or ankle joints, and a study indicating that changing the angle of foot progression can reduce the pressure applied to the knee during stair walking in patients with knee arthritis was reported [11]. In addition, a study reported that even in the case normal adults, changes in the angle of foot progression during stair walking made significant changes in the hip and knee joints and affected the ground reaction force thereby reducing the load on the knee joint, and effectively preventing knee osteoarthritis [12].

As such, many of studies on stair walking and changes in the angle of foot progression focused on knee arthritis patients or general normal people but recently, studies regarding the muscle activity of the overall lower extremity muscles including the ankle joints and the movement of the center of pressure in people with chronic ankle instability have been reported [13], [14]. However, as described above, studies examining changes in the center of pressure according to changes in the angle of foot progression during stair walking, which is the most frequent next to flatland walking, are insufficient.

\section{METHODS}

\section{A. Participants}

This study selected 20 adult men and women aged at least 25 years but less than 35 years with chronic ankle instability among the employees of the Daegu Hospital of the Korea Workers' Compensation and Welfare Service located in Daegu Metropolitan City. The criteria for the selection of subjects with chronic ankle instability were those who felt wobbling in the ankle joint and scored not higher than 24 points in a test using the Cumberland Ankle Instability Tool [15]. A written consent to participate in the experiment was distributed to all the study subjects to obtain voluntary content to participate after explaining the purpose of this study and the contents of the experiment. This study was conducted after obtaining approval from the Daegu University Bioethics Committee (Approval: 1040621202004-HR-012). The general characteristics of the subjects who participated in this study are as shown in Table 1.

Table 1. General characteristics of subjects

\section{B. Procedures}

When the subjects go up the stairs, the angle of foot progression vertical to the ground was set as the neutral posture, that turned $15^{\circ} \sim 20^{\circ}$ medially was set as the toein posture, and that turned laterally as the toe-out posture. To prevent the angle of the foot from changing during the experiment, the angle was marked with tape at each step of the stairs. In addition, Wii Balance Boards were placed on the top and bottom steps during stair walking to measure changes in the center of pressure.

The researcher practiced the experiment in advance to be fully aware of matters that require attention. Before proceeding with the main experiment, the researcher first showed the demonstration to make the subjects recognize the correct postures so that the subjects could carry out the experiment. While the subjects were carrying out the experiment, the researcher checked whether the subjects were carrying out the experiment in the correct postures.

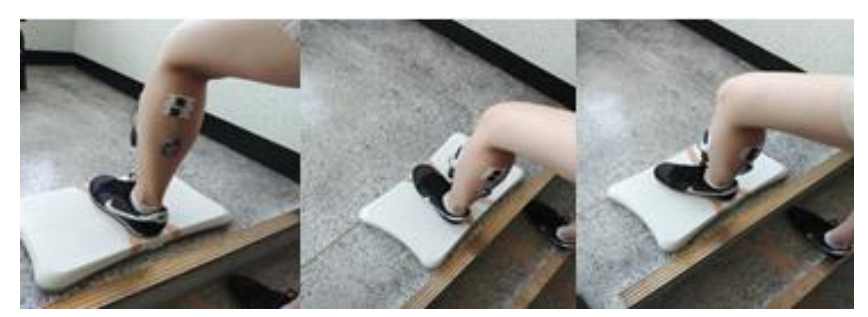

Figure 1. Ankle posture (neutral, toe-in, toe-out)

\section{Assessment}

The Wii Balance Board (Nintendo, WA, USA) is a home game device developed by Nintendo, which is widely distributed at a relatively low price, and enables the measurement of the center of pressure and weight because an analog pressure sensor is attached to the corner of its square plate. A previous study [16] extracted the measured values of the center of pressure in normal persons from the pressure sensor of the Wii Balance Board, and evaluated the reliability by comparing the foregoing measured values with values measured on a force plate. In the results, the intraclass correlation coefficient (ICC) value of the Wii Balance Board and the force plate showed high reliability with interrater reliability of $0.89 \sim 0.79$ and intra-rater of $0.92 \sim 0.70$. 
Table 2. Changes in COP during stair ascending

\begin{tabular}{lcccccc}
\hline \multicolumn{1}{c}{ COP } & Neutral & Toe-out & Toe-in & F & $p$ & Effect size(f) \\
\hline $\begin{array}{l}\text { Medial/lateral } \\
\text { moving distance }\end{array}$ & $8211.38 \pm 5612.35$ & $14081.1 \pm 14782.2$ & $6098.52 \pm 2095.15$ & 4.035 & $.023^{*}$ & -0.274 \\
$\begin{array}{l}\text { Medial/lateral } \\
\text { moving ranges }\end{array}$ & $1417.86 \pm 1711.24$ & $1662.97 \pm 2189.53$ & $395.81 \pm 212.84$ & 3.489 & $.037^{*}$ & -0.356 \\
Average moving & $1252.03 \pm 1462.35$ & $1361.59 \pm 1823.93$ & $777.87 \pm 240.93$ & 1.046 & .358 & -0.195 \\
velocity & & & & & & \\
Anterior/posterior & $6416.59 \pm 7652.93$ & $6911.72 \pm 9601.03$ & $3726.94 \pm 1486.52$ & 1.152 & .323 & -0.206 \\
moving distance & & & & & & \\
Total travel range & $12520.36 \pm 14623.5$ & $13616.1 \pm 18238.9$ & $7778.78 \pm 2409.33$ & 1.046 & .358 & -0.195 \\
& & & & & & \\
Total moving range & $6267.64 \pm 8630.3$ & $7505.62 \pm 1.77$ & $3007.84 \pm 2253.44$ & .819 & .446 & -0.169 \\
\hline
\end{tabular}

${ }^{\mathrm{a}}$ mean \pm standard deviation

${ }^{*} \mathrm{p}<.05$

Therefore, in this study, the Wii Balance Board was used to measure the total travel range, moving range, and average velocity, subdivide the total travel range into that along the horizontal axis and that along the vertical axis, and measure the foregoing, respectively, and Balancia software ver. 2.0 (Mintosystem, Seoul, Korea) was used as a program to analyze the measured data. The ground reaction force applied while the foot was in contact with the ground was extracted by sampling at $100 \mathrm{~Hz}$ per second, and the resultant values in the stored data were analyzed using Excel software.

\section{STATISTICAL ANALYSIS}

The Kolmogorov-Smirnov test was used for a normal distribution. One-way ANOVA was conducted to compare and analyze the effects of the changes in the angle of foot progression and LSD post-tests were conducted to find out differences according to the angles of progression. Statistical analyses were performed using SPSS (Statistical package for the social sciences) version 20.0 for Window software (SPSS, IBM, USA), and the statistical significance level was set at $\alpha=.05$. The effect size f-test was conducted which is a standardized indicator of the difference between groups.

\section{RESULTS}

The total moving distance, moving ranges, and average velocities of the COP during stair ascending according to changes in the angle of foot progression(neutral, toe-in, toeout) were compared, the total travel range was subdivided into anterior/posterior and medial/lateral ones, and moving ranges were also checked for medial and lateral movements, which show statistically significant differences. In the results, there was no statistically significant difference in the resultant values of the total moving distance, moving ranges, average velocities, or anterior/posterior movements of the COP among ankle neutral, toe-in, and toe-out postures $(p>.05)$. but significant differences appeared in the medial/lateral moving distance and moving ranges of the COP between toe-in and toe-out postures $(\mathrm{p}<.05)$ (Table 2) (Figure 2)

The effect sizes for all items measured during stair ascending were smaller in the toe-in posture than in the ankle neutral and toe-out postures $(\mathrm{f}<0.25)$ (Table 2$)$

\section{DISCUSSION}

A high degree of ankle instability negatively affects healthrelated quality of life and makes the relevant person experience more functional limitations in daily life [17]. In addition, since the feet and the ankles provide weight support during physical activities and stability and flexibility during movements, various functions of the foot can be evaluated by measuring changes in the COP and the size and distribution of the COP are affected by pain, foot structures, or functional conditions [18]. This study checked changes in the COP in 20 adults while applying different angles of foot progression during stair ascending.

There was no statistically significant difference in the total 
moving distance, moving ranges, average velocities, or anterior/posterior movements of the COP among ankle neutral, tor-out, and toe-in postures but significant differences appeared in the medial/lateral moving distance and moving ranges of the COP between toe-out and toe-in postures. No significant difference appeared in the comparison between toe-in and neutral postures and between toe-out and neutral postures.

A previous study stated that the movement path of the COP shows the movement of the pressure distribution according to the movement of the foot during the gait cycle and that the anterior/posterior movement range of the COP reflects the degree of progression of the COP while the left/rightward movement range means medial/lateral instability [19]. Another previous study reported that in the case of subjects with ankle instability, the section velocity decreased in the anterior/posterior and medial/lateral directions during dynamic postural control [20], and still another previous study indicated that in the case of subjects with ankle instability, the section velocity and direction control ability decreased in the medial/lateral direction, not in the anterior/posterior direction so that left/rightward sways appeared during rhythmic weight shifts such as flatland walking and stair walking and that the COP showed a tendency to be deviated laterally [21]. In addition, a study reported that the weakening of proprioceptive sensibility tends to deteriorate the sensation of the sole of the foot to make the COP deviate anteriorly and laterally from the foot and negatively affects the average pressure and dynamic movements [22], and another study reported that the movements of inversion and lateral rotation of the ankle joint increase during walking leading to the deterioration of the stability of postures so that large body shaking appears [20].

It is thought that the stability of the ankle increased in the toe-in posture during stair ascending because a posture similar to the pronation described in the previous study [23] was made leading to the intorsion of the axes of the knee joint and hip joint and overall increases in the force on the structure in the medial plane of the lower limb so that the medial/lateral moving distance and moving ranges of the COP decreased during rhythmic weight shift and postural control.

This study had difficulties in objectification because the experiment was carried out with a small number of subjects when changes in COP were measured and there were errors due to differences in the body structures of individuals and due to acquired habits. Therefore, it is considered that studies hereafter should compare between men and women with chronic ankle instability during overall stair walking, including ascending as well as going down the stairs and should subdivide changes in the COP in persons at various ages and in various occupational groups more into many items and objectify the items.
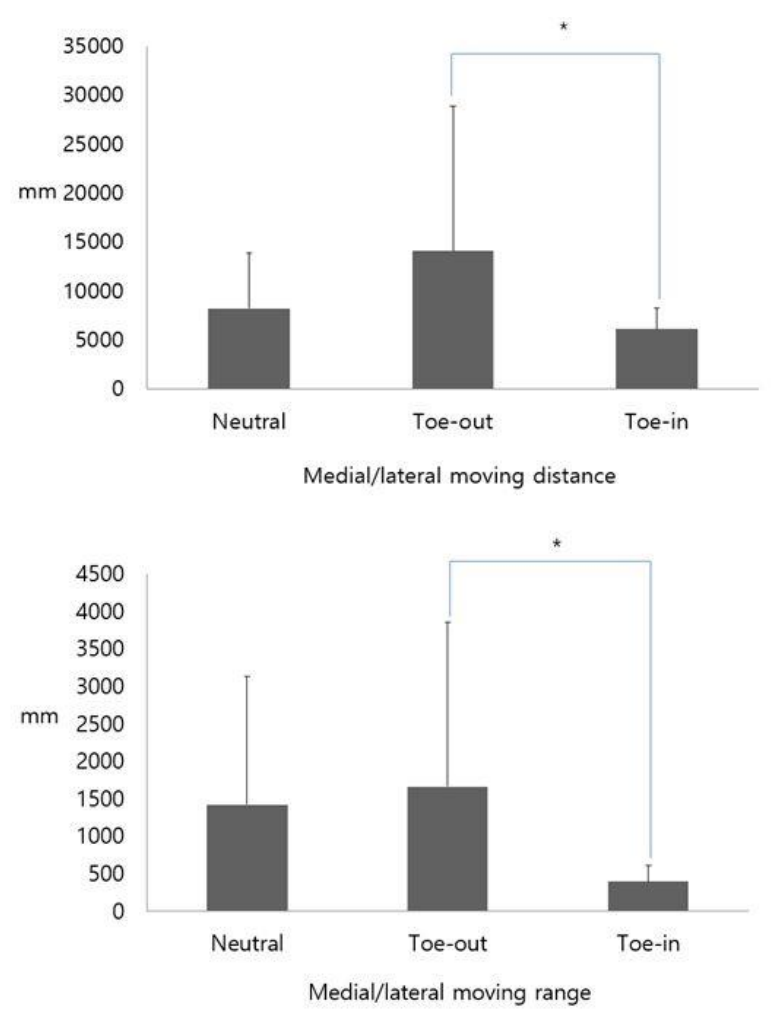

Figure 2. Mediolateral moving distance \& moving ranges

\section{CONCLUSION}

The purpose of this study was to figure out the effects of different angles of foot progression applied to stair ascending on changes in the COP in adults with chronic ankle instability. In the results of this study, medial/lateral movements of the center of pressure showed significant differences between the toe-in and toe-out postures.

It could be seen that when adults with chronic ankle instability climb the stairs, the toe-in posture reduces the medial/lateral movements of the center of pressure thereby increasing the stability of the ankle and effectively preventing re-injuries. It is expected that the findings of this study will be utilized as a reference by adults with chronic ankle instability during their stair walking.

\section{REFERENCE}

[1] D. T. Fong, Y. Hong, L. K. Chan, P. S. Yung, K. M. Chan, "A systematic review on ankle injury and ankle sprain in sports," Sports Med, 37(1):73-94, 2007.

[2] A. Leardini, J. J. O'Connor, S. Giannini, "Biomechanics of the natural, arthritic, and replaced human ankle joint," J Foot Ankle Res, 7(1):8, 2014.

[3] B. Hintermann, "Biomechanics of the unstable ankle joint and clinical implications," Med Sci Sports Exerc, 31(7 Suppl):S459-S469, 1999

[4] T. J. Hubbard, J. Hertel, "Anterior positional fault of the fibula after sub-acute lateral ankle sprains," Man Ther, 13(1):63-67, 2008. 
[5] P. A. Gribble, E. Delahunt, C. M. Bleakley, "Selection criteria for patients with chronic ankle instability in controlled research: a position statement of the International Ankle Consortium," J Athl Train, 49(1):121-127, 2014.

[6] J. Hertel, "Functional Anatomy, Pathomechanics, and Pathophysiology of Lateral Ankle Instability," J Athl Train, 37(4):364-375, 2002.

[7] D. R. Brown, Y. Wang, A. Ward, " Chronic psychological effects of exercise and exercise plus cognitive strategies," Med Sci Sports Exerc, 27(5):765775, 1995.

[8] M. S. Roys, "Serious stair injuries can be prevented by improved stair design," Appl Ergon, 32(2):135-139, 2001.

[9] T. P. Andriacchi, G. B. Andersson, R. W. Fermier, D. Stern, J. O. Galante, “A study of lower-limb mechanics during stair-ascending, "J Bone Joint Surg Am, 62(5):749-757, 1980.

[10] A. Protopapadaki, W. I. Drechsler, M. C. Cramp, F. J. Coutts, O. M. Scott, "Hip, knee, ankle kinematics and kinetics during stair ascent and descent in healthy young individuals," Clin Biomech (Bristol, Avon), 22(2):203-210, 2007.

[11] S. Wang, K. H. C. Chan, R. H. M. Lam, "Effects of foot progression angle adjustment on external knee adduction moment and knee adduction angular impulse during stair ascent and descent," Hum Mov Sci, 64:213220, 2019.

[12] R. Tsukagoshi, M. Goto, H. Senoo, R. Honda, "Influence of foot progression angle on knee adduction and flexion moment during stair ascending in healthy individuals," Gait Posture, 71:163-169, 2019.

[13] R. M. Koldenhoven, M. A. Feger, J. J. Fraser, S. Saliba, J. Hertel, "Surface electromyography and plantar pressure during walking in young adults with chronic ankle instability, " Knee Surg Sports Traumatol Arthrosc, 24(4):1060-1070, 2016.
[14] M. A. Feger, L. Donovan, J. M. Hart, J. Hertel, "Lower extremity muscle activation in patients with or without chronic ankle instability during walking," J Athl Train, 50(4):350-357, 2015.

[15] K Friel, N. McLean, C. Myers, M. Caceres, "Ipsilateral hip abductor weakness after inversion ankle sprain," $J$ Athl Train, 41(1):74-78, 2006.

[16] D. S. Park, D. Y. Lee, S. J. Choi, W. S. Shin, "Reliability and validity of the balancia using wii balance board for assessment of balance with stroke patients," Journal of the Korea Academia-Industrial cooperation Society, 14(6):2767-2772, 2013.

[17] B. L. Arnold, C. J. Wright, S. E. Ross, "Functional ankle instability and health-related quality of life," $J$ Athl Train, 46(6):634-641, 2011.

[18] C. J. Sneyers, R. Lysens, H. Feys, R. Andries, "Influence of malalignment of feet on the plantar pressure pattern in running, " Foot Ankle Int, 16(10):624-632, 1995.

[19] C. R. Young, "The F-SCAN system of foot pressure analysis," Clin Podiatr Med Surg, 10(3):455-461, 1993.

[20] P. A. Gribble, J. Hertel, "Effect of lower-extremity muscle fatigue on postural control," Arch Phys Med Rehabil, 85(4):589-592, 2004.

[21] C. Brown, B. Bowser, K. J. Simpson, "Movement variability during single leg jump landings in individuals with and without chronic ankle instability," Clin Biomech (Bristol, Avon), 27(1):52-63, 2012.

[22] H. Schmidt, L. D. Sauer, S. Y. Lee, S. Saliba, J. Hertel, "Increased in-shoe lateral plantar pressures with chronic ankle instability," Foot Ankle Int, 32(11):1075-1080, 2011.

[23] G. Kolt, "The malalignment syndrome: implications for medicine and sport," British Association of Sport and Excercise Medicine, 2004.

\section{Creative Commons Attribution License 4.0 (Attribution 4.0 International, CC BY 4.0)}

This article is published under the terms of the Creative Commons Attribution License 4.0 https://creativecommons.org/licenses/by/4.0/deed.en_US 